\title{
Histoplasmosis with Deep CNS Involvement: Case Presentation with Discussion and Literature Review
}

\author{
Omid R. Hariri ${ }^{1}$ Tanya Minasian ${ }^{1}$ Syed A. Quadri ${ }^{1}$ Anya Dyurgerova ${ }^{2} \quad$ Saman Farr $^{2}$ Dan E. Miulli ${ }^{1}$ \\ Javed Siddiqi ${ }^{1}$
}

${ }^{1}$ Department of Neurosurgery, Arrowhead Regional Medical Center, Colton, California, United States

2 Division of Neurosurgery, Department of Surgery, Western University of Health Sciences, Pomona, California, United States

\begin{abstract}
Address for correspondence Omid R. Hariri, DO, MSc, Department of Neurosurgery, Arrowhead Regional Medical Center, 400 N. Pepper Avenue, Colton, CA 92324, United States (e-mail: ohaririucla@gmail.com).
\end{abstract}

J Neurol Surg Rep 2015;76:e167-e172.

\section{Introduction}

Histoplasmosis, an infection caused by the dimorphic fungus Histoplasma capsulatum, is considered one of the most common fungal respiratory infections in the world. It is endemic in the central and southeastern states of the United States, Latin America, Africa, and now parts of Asia with an estimated 40 million people in the United States infected. ${ }^{1}$

H. capsulatum is a dimorphic fungus taking both mycelia and yeast forms. It feeds on nitrogen-rich soils, especially those replete with bird and bat droppings. When inhabited soils like these are disturbed, spores, which are the reproducing body of the mycelia, can become airborne and inhaled. Once they are inhaled, infection begins. Due to the elevated temperature inside the lungs compared with room air, the spores grow as unicellular haploid yeast cells and are phagocytosed by macrophages. The fungus continues to live within the phagolysosomes of the macrophages where it increases the $\mathrm{pH}$ and potentially disturbs intracellular processes. ${ }^{2,3}$
License terms

Stuttgart · New York

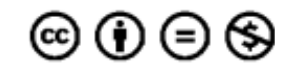

(c) 2015 Georg Thieme Verlag KG received

December 9, 2014

accepted

April 6, 2015

published online

June 26, 2015
DOI http://dx.doi.org/

10.1055/s-0035-1554932. ISSN 2193-6366. 
Of the documented histoplasmosis infections in immunocompetent individuals, 50 to $90 \%$ are asymptomatic, and of the symptomatic infections, $80 \%$ require no therapy and are self-resolving. ${ }^{4}$ Conversely, some patients develop a progressive extrapulmonary infection, or rather a disseminated form of histoplasmosis where the infection spreads beyond its origin into other parts of the body. The symptomatic cases of infection are frequently found in immunocompromised patients, especially in individuals with acquired immunodeficiency virus (AIDS), those with ventriculoperitoneal shunts, ${ }^{5}$ recipients of transplants, or in patients taking corticosteroids and tumor necrosis factor- $\alpha$ antagonists. ${ }^{4}$

In particular, 3 to $5 \%$ of AIDS patients within endemic areas of North America develop disseminated histoplasmosis. ${ }^{6} \mathrm{~A}$ prospective study correlated that as an individual's CD4+ count decreases, the risk of acquiring histoplasmosis increases. $^{7}$ Of these 3 to $5 \%$ infected immunocompromised patients, $90 \%$ have infections that advance into progressive disseminated histoplasmosis (PDH). Of all the patients with $\mathrm{PDH}, 10$ to $20 \%$ develop central nervous system (CNS) involvement ${ }^{4}$ that affects the meninges, the spinal cord, and/or the brain. ${ }^{8}$ Patients with a CNS infection have a mortality rate of 20 to $40 \%$ and a relapse rate of $50 \%{ }^{9}$

Even though the incidence of CNS histoplasmosis in nonendemic areas is increasing, the diagnosis of isolated CNS histoplasmosis in these areas remains a challenge for clinicians for a number of reasons. CNS histoplasmosis is difficult to diagnose, especially in geographic areas that are not endemic, because it is often overlooked or mistaken for other pathologies due to its nonspecific symptom set. It may include all, some, or none of the following signs: acute and chronic meningitis, stroke due to infected emboli, diffuse encephalitis, ring-enhancing lesions, neurologic deficits, chronic recurrent hydrocephalus, elevated cerebrospinal fluid (CSF) protein, and an elevated CSF white blood cell count. ${ }^{4,10-12}$ Although these signs are indicative of localized CNS histoplasmosis, addition of systemic symptoms, such as a fever or weight loss, ${ }^{13}$ may indicate a disseminated disease.

Failure to diagnose CNS histoplasmosis quickly leads to a prolonged infection and lowers the chance of recovery. The following is a case presentation, discussion, and literature review on disseminated histoplasmosis with CNS involvement in an immunocompromised patient diagnosed outside of the endemic regions.

\section{Case Study}

A 32-year-old Hispanic man with advanced AIDS (CD4+ count: $31 ; 290,000$ copies of human immunodeficiency virus RNA) presented with an altered mental status and reported confusion for the past 3 months. He had refused to continue highly active antiretroviral therapy 1 year prior. The patient complained of losing 30 pounds in 3 months and a persistent cough for 3 weeks. The patient's family disclosed that he had a recent fever and chills. The patient had also been admitted 3 weeks prior due to abdominal pain. He had a history of incarceration and deportation. He admitted to tobacco, alcohol, and polysubstance abuse (mainly methamphetamines), but he denied any use in the past year. The patient was previously employed as a truck driver, and it cannot be ruled out that he may have visited and been exposed to endemic regions of histoplasmosis.

On physical examination, fever was confirmed with a temperature of $101.7^{\circ} \mathrm{F}$. The patient was diaphoretic, cachectic, somnolent, and lethargic. Bilateral radial and dorsalis pedis pulses were $+2 / 4$. The patient's consciousness level was Glasgow Coma Scale 12 (Motor 6, Verbal 2, Eyes 4) with repetitive nonfluent speech. He had a disconjugate gaze with a right gaze preference and symmetrical movement of his extremities but with poor effort. No pronator drift was noted, and the patient's sensation was grossly intact. Deep tendon reflexes were $+2 / 4$ in all extremities. The patient's pupils were equal, round, and reactive to light, and his extraocular movements were intact without nystagmus. The patient's cranial nerves II-XII were grossly intact, and he showed no cerebellar deficits.

Laboratory results such as acid-fast bacilli smears, purified protein derivative, and QuantiFERON Gold were negative for tuberculosis. Blood cultures and CSF cultures showed no growth. CSF studies showed leukocytes (white blood cells) $1 / \mu \mathrm{L}$, red blood cells $1,000 / \mu \mathrm{L}$, lymphocytes $100 / \mu \mathrm{L}$, glucose $48 \mathrm{ng} / \mathrm{dL}$, and total protein $134 \mathrm{mg} / \mathrm{dL}$.

Lung computed tomography (CT) findings indicated a pulmonary histoplasmosis infection. Furthermore, magnetic resonance imaging (MRI) of the brain revealed a ring-enhancing lesion in the left caudate nucleus (-Fig. 1A). There were no true signs of meningitis on MRI. Given the extensive mass effect and cerebral edema, Decadron was started, as well as seizure prophylaxis with Keppra. A left retroperitoneal node biopsy was performed and indicated a benign inflammatory process with organisms compatible with fungal yeast, strongly suggesting histoplasmosis. Once a diagnosis of disseminated disease with CNS involvement was made, the patient was placed on amphotericin and itraconazole and taken off of Decadron, but Keppra was continued.

Throughout the hospital course, there was clear neurologic improvement, and the patient was discharged 3 weeks later in stable condition. On discharge, he was able to converse, ambulate without difficulty, and adequately perform activities of daily living with assistance. The patient was sent home with instructions to follow up and continue with lifelong antifungal treatment. Six weeks after the initial diagnosis, the patient's left basal ganglia mass significantly decreased from $30 \times 36.9 \mathrm{~mm}$ to $28.3 \times 23.3 \mathrm{~mm}$ (-Fig. 1B). Cerebral edema also diminished.

\section{Discussion}

Due to its mode of transmission via spore inhalation, histoplasmosis most commonly manifests in the lungs, ranging from pneumonitis to severe acute respiratory distress syndrome. The yeast form is disseminated via macrophages as they travel throughout the body.$^{14}$ Individuals with impaired cell-mediated immunity lack the ability to eliminate the intracellular pathogen, which 


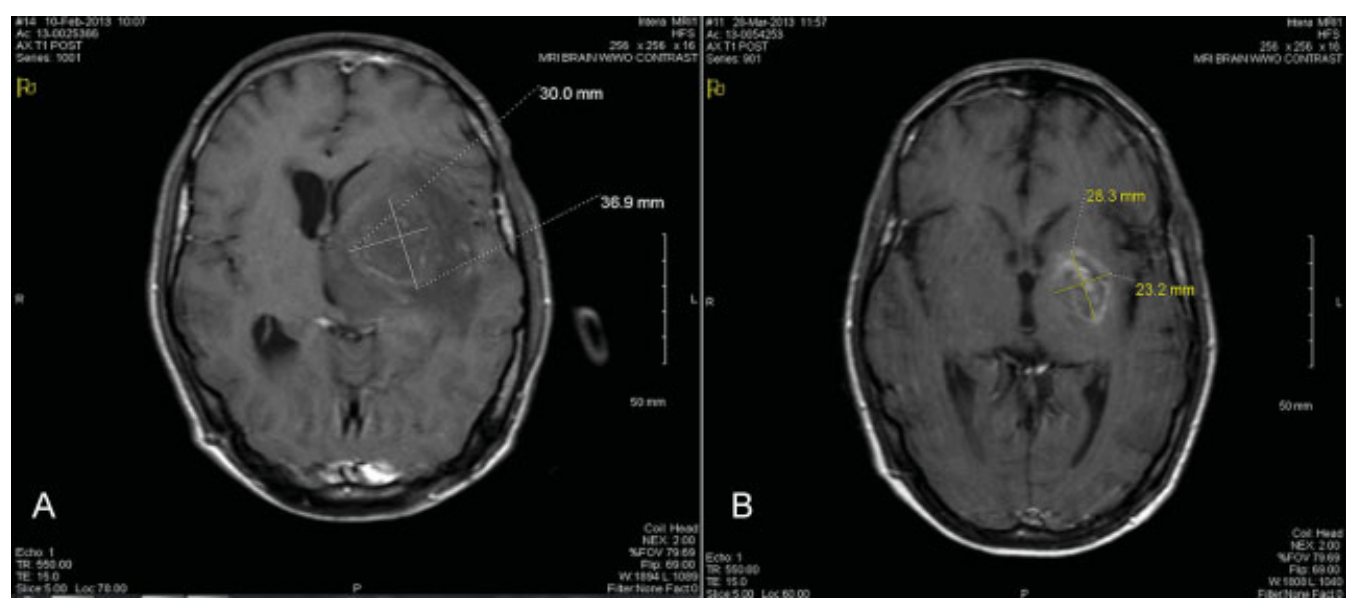

Fig. 1 (A) Pretreatment T1-weighted magnetic resonance imaging (MRI) with gadolinium. Axial image showing left basal ganglia mass of $30 \times 36.9 \mathrm{~mm}$. (B) T1-weighted MRI with gadolinium 6 weeks after treatment was initiated. Axial image showing decrease in left basal ganglia mass to $28.3 \times 23.3 \mathrm{~mm}$.

places them at greater risk for symptomatic histoplasmosis and disseminated disease.

The mechanism of the hematogenous dissemination of the infection to the meninges or brain is not well understood, and there have been very few studies done on this subject. A murine model indicates that $H$. capsulatum protein Yps3p interacts with microglial cells in the CNS, leading to activation of the nuclear factor-кB via a signaling pathway involving a pathogen recognition receptor on microglial cells, toll-like receptor 2. This stimulates expression of a proinflammatory immune mediator, chemokine (C-C motif) ligand 2 in microglia. ${ }^{15}$

CNS histoplasmosis can present with or without pulmonary involvement; some isolated cases with absence of extraneural signs and symptoms have been reported. ${ }^{10-12} \mathrm{~A}$ study found that the most common symptoms of CNS involvement in PDH are acute or chronic meningitis, a lowered level of consciousness $(28.8 \%)$, headache (24\%), cranial nerve deficiency (19.2\%), seizures (13.5\%), and confusion. ${ }^{8}$ However, other symptoms are highly diverse among individual cases, and cerebral involvement is only clinically evident in 10 to $20 \%$ of patients. ${ }^{8}$

In rare cases, patients with CNS histoplasmosis may present with myelopathy (cervical and thoracic), hydrocephalus (chronic recurrent), or cachexia and hypercalcemia. ${ }^{11,12,16}$ Hydrocephalus is one of the significant complications of this condition that may possibly be identified even before meningitis is diagnosed. ${ }^{12}$

In immunocompetent individuals, $H$. capsulatum infection has been diagnosed in unusual stroke cases ${ }^{10,11,17-19}$ or mistaken for a brain tumor. ${ }^{20} \mathrm{~A}$ case with a patient experiencing short-term memory loss and cognitive impairment was also reported. ${ }^{21}$ In our case the patient along with confusion and altered mental status had speech and gaze problems. All possible symptoms reported in the literature at the time of presentation were collated in $\mathbf{- T a b l e} \mathbf{1}$.

Focal CNS infection is also possible but even rarer. An autopsy study showed that the rate of dissemination to the
CNS may be higher than diagnosed and may persist without symptoms, suggesting that focal CNS infection could either be primary infections or manifestations of latent infections. ${ }^{22}$ $H$. capsulatum can remain in the body undetected for years before it becomes symptomatic; furthermore, it can relapse with a delayed onset of symptoms. This hypothesis is supported by a case of a patient with advanced AIDS and previous disseminated histoplasmosis. The patient presented with a focal CNS infection 12 years after he had been diagnosed with and treated for disseminated histoplasmosis. ${ }^{23}$ In another case, an immunocompetent woman in Arizona presented with slight cognitive deficits and seizures 2 years after she had undergone treatment for a gastrointestinal histoplasmosis infection. She was diagnosed with an intramedullary histoplasmosis spinal cord abscess secondary to a relapse of the previous gastrointestinal infection. ${ }^{24}$

MRIs often show single or multiple ring-enhancing lesions in CNS histoplasmosis cases ${ }^{13,16,20,23-29}$ as presented here. However, ring-enhancing lesions can also be indicative of an abscess, a necrotic tumor, ${ }^{30}$ subdural and epidural empyema, ${ }^{31}$ or toxoplasmosis. ${ }^{27,31}$ Histoplasmosis should be considered if symptoms correlate to that of the infection or if the patient has had exposure to an endemic area.

Multiple tests for diagnosis of $H$. capsulatum in the CNS are necessary due to the variability in symptoms and the number of false-negative tests. Culturing of the CSF or CNS parenchymal tissue is the gold standard for diagnosis of CNS histoplasmosis. Antigen detection in CSF culture has a sensitivity of $38 \%$ and a specificity of $98 \%{ }^{9}{ }^{9}$ Additional tests for $\mathrm{H}$. capsulatum polysaccharide antigen (HPA) in CSF, urine, or serum can be performed to aid in the diagnosis and monitor response to therapy. The sensitivity of these is 38 to $67 \%$, $71 \%$, and $38 \%$, respectively. ${ }^{8}$ Antibody testing in CSF and serum has 80 to $89 \%$ and $92 \%$ sensitivity, respectively, although these tests are susceptible to cross-reactivity leading to false positives. Serologic testing is especially difficult with AIDS patients and often provides false negatives even with an active infection. ${ }^{8}$ In addition, positive CSF culture results are 
Table 1 Symptoms, diagnostic techniques, and treatment reported/suggested in the literature ${ }^{\text {a }}$

\begin{tabular}{|c|c|}
\hline \multicolumn{2}{|c|}{ Symptoms/Possible Presentations Reported in the Literature } \\
\hline \multicolumn{2}{|l|}{\begin{tabular}{l|l} 
Acute meningitis & \\
\end{tabular}} \\
\hline Chronic Meningitis & $\begin{array}{l}\text { Undiagnosed up to } 10 \text { y because tests for other causes } \\
\text { were negative has been reported. }{ }^{32} \text { It should always be } \\
\text { included in the differential diagnoses of chronic meningitis } \\
\text { in nonendemic areas even in the case of nonimmunocompromised } \\
\text { individuals }\end{array}$ \\
\hline Stroke & Recurrent strokes due to infected emboli ${ }^{20,31,34}$ \\
\hline \multicolumn{2}{|l|}{ Seizures } \\
\hline \multicolumn{2}{|l|}{ Headache } \\
\hline Focal parenchymal lesions of the brain or spinal cord & Ring-enhancing lesions in brain \\
\hline \multicolumn{2}{|l|}{ Diffuse encephalitis } \\
\hline Hydrocephalus & $\begin{array}{l}\text { It is an important complications of CNS histoplasmosis that } \\
\text { may be identified even before meningitis is diagnosed. }{ }^{32} \\
\text { Chronic recurrent hydrocephalus can occur }\end{array}$ \\
\hline Myelopathy & Cervical and thoracic myelopathy ${ }^{31}$ \\
\hline \multicolumn{2}{|l|}{ Fever or weight loss } \\
\hline Neurologic deficits & $\begin{array}{l}\text { Repetitive nonfluent speech, disconjugate gaze with a right } \\
\text { gaze preference }\end{array}$ \\
\hline Memory loss & $\begin{array}{l}\text { Short-term memory, difficulty recalling names, retaining } \\
\text { information while reading and getting lost while driving }\end{array}$ \\
\hline Cognitive impairment & $\begin{array}{l}\text { Word-finding problems and difficulty organizing, making } \\
\text { decisions, and slowed processing speed }\end{array}$ \\
\hline Absence of extraneural signs and symptoms & $\begin{array}{l}\text { Isolated cases of CNS histoplasmosis with absence of } \\
\text { extraneural signs and symptoms have been reported }\end{array}$ \\
\hline \multicolumn{2}{|c|}{ Diagnostic and Imaging Techniques Reported in the Literature } \\
\hline \multicolumn{2}{|l|}{ Elevated CSF WBC } \\
\hline \multicolumn{2}{|l|}{ Elevated CSF protein } \\
\hline \multicolumn{2}{|l|}{ Decreased CSF glucose } \\
\hline Immunity level & $\begin{array}{l}\text { Although very rare in immunocompetent patients, cases of } \\
\text { histoplasmosis have been reported } 15,18,20,31,34\end{array}$ \\
\hline Culture & $\begin{array}{l}\text { Culture of } H \text {. capsulatum from CSF, brain tissue, or other } \\
\text { sites is the gold standard for diagnosis }\end{array}$ \\
\hline Culture-negative cases & $\begin{array}{l}\text { CSF culture could be negative. }{ }^{19, \text { our case }} \text { If culture is negative, } \\
\text { then in those cases detection of } H \text {. capsulatum antigen in CSF, } \\
\text { urine, or blood is helpful diagnostically }\end{array}$ \\
\hline False-positive tests & $\begin{array}{l}\text { It should be noted that antigen or serologic tests may also show } \\
\text { false-positive results because of cross reactions due to infections } \\
\text { by other fungi } 32\end{array}$ \\
\hline Low sensitivity & $\begin{array}{l}\text { It is essential to do repeated cultures with a large volume of CSF } \\
\text { because of the low sensitivity of the culture. It is recommended } \\
\text { that tests for CNS histoplasmosis be repeated with large } \\
\text { volumes of CSF even if negative results are } \\
\text { obtained initially } 32 \text {, our case }\end{array}$ \\
\hline Neuroendoscopic examination and biopsy & $\begin{array}{l}\text { A biopsy of the floor of the third ventricle and the subarachnoid } \\
\text { space, and the collection of ventricular CSF contributed to the } \\
\text { correct diagnoses of histoplasmosis after CSF cultures and CSF } \\
\text { antibody tests were negative }{ }^{12,19}\end{array}$ \\
\hline CT-guided biopsy of a retroperitoneal lymph node & $\begin{array}{l}\text { CT-guided biopsy of a retroperitoneal lymph node yielded the } \\
\text { results with much less morbidity than a left-sided dominant } \\
\text { hemisphere deep basal ganglia lesion biopsy would have [our case] }\end{array}$ \\
\hline
\end{tabular}


Table 1 (Continued)

\begin{tabular}{|l|l|}
\hline Liposomal amphotericin B & Initial course $20,21,31,32$, our case \\
\hline Itraconazole & $\begin{array}{l}\text { For } 1 \text { y after the initial course of liposomal } \\
\text { amphotericin } \mathrm{B}^{20,21,31,32, \text { our case }}\end{array}$ \\
\hline Fluconazole & $20,21,31,32$ \\
\hline Newer azoles & $\begin{array}{l}\text { Newer azoles such as voriconazole have been shown to be } \\
\text { effective in both immunocompromised and } \\
\text { immunocompetent hosts }\end{array}$ \\
\hline
\end{tabular}

Abbreviations: CNS, central nervous system; CSF, cerebrospinal fluid; CT, computed tomography; WBC, white blood cells.

${ }^{a}$ It should be kept in mind that patients presenting with these symptoms can be immunocompromised or immunocompetent belonging to endemic or nonendemic areas with or without any extraneural signs and symptoms.

similar to results seen in other fungal and tuberculous meningitis. ${ }^{1}$ Therefore, other tests must be performed.

Multiple antigen and culture tests must be performed given the low sensitivities of each of the individual tests. A recent study found variability between spinal and ventricular CSF histoplasmosis antibody test results. Rangel-Castilla et al described a case of CNS histoplasmosis with basilar arachnoiditis where CSF cultures and CSF antibody tests were negative. To diagnose the patient, a neuroendoscopic examination of the ventricles and basal cisterns was done. A biopsy of the floor of the third ventricle and the subarachnoid space, and the collection of ventricular CSF contributed to the correct diagnoses of histoplasmosis. ${ }^{32}$ The antibodies were only identifiable in the ventricular CSF. It was hypothesized that the differences observed were a sequela of compartmentalization of the subarachnoid space caused by arachnoid membrane scarring as a result of an inflammatory process. ${ }^{32}$ Similarly, in another study, a diagnosis was made only after testing the patient's ventricular CSF 5 months after the infection started, making the recovery much more arduous. ${ }^{26}$

Similarly, histoplasmosis remained undetected in the present case until a more aggressive diagnostic approach was performed. In our case a CT-guided biopsy of a retroperitoneal lymph node yielded the results with much less morbidity than a left-sided dominant hemisphere deep basal ganglia lesion biopsy would have. A correct diagnosis was procured despite the negative CSF cultures, urine, and blood tests, and it decreased the risk of possible complications accompanying a brain biopsy.

In most CNS histoplasmosis cases, one or a combination of any three antifungals, amphotericin B, fluconazole, and itraconazole, have been used. Although no prospective studies of an optimal treatment regimen for CNS histoplasmosis are available, numerous case reports have documented variable success of initial treatment with amphotericin B followed by a triazole (e.g., fluconazole, itraconazole, or voriconazole). ${ }^{10-12,29}$ Newer azoles such as voriconazole are currently being studied for efficacy in CNS histoplasmosis and may be shown to provide more consistent success in the future. ${ }^{33}$

Liposomal amphotericin B is preferred over standard amphotericin B formulation for its greater CNS penetration and lower toxicity. The Infectious Diseases Society of America's 2007 Guidelines for Management of Histoplasmosis recommend $5.0 \mathrm{mg} / \mathrm{kg}$ of liposomal amphotericin B for 4 to 6 weeks. This should be followed by itraconazole for at least 12 months until CSF findings and HPA are negative for immunocompetent patients. ${ }^{34}$ In immunosuppressed patients, such as the present case, lifelong antifungal therapy may be needed to prevent relapse.

\section{Conclusion}

This case, along with numerous other published cases, illustrates that CNS histoplasmosis can present with a wide array of signs and symptoms with or without extraneural involvement even in nonendemic areas irrespective of the patient's immunologic status, making it more difficult for clinicians to diagnose. However clinical recovery from CNS histoplasmosis is possible with early recognition of symptoms and proper steps toward a correct diagnosis. An aggressive regime of more invasive testing may be required.

Treatment with amphotericin B followed by itraconazole proved effective in mitigating associated CNS lesions and resolving neurologic deficits. This case demonstrates that with aggressive medical management, treating intracranial deep mass lesions is possible, and that disseminated histoplasmosis with CNS involvement can be correctly diagnosed and treated, despite negative CSF and serology studies. The critical step in ensuring survival in patients with a CNS histoplasmosis infection is a quick diagnosis. Therefore, histoplasmosis should be considered in cases of chronic meningitis, focal brain lesions, recurrent hydrocephalus, and unexplained neurologic symptoms.

\section{References}

1 Kauffman CA. Histoplasmosis: a clinical and laboratory update. Clin Microbiol Rev 2007;20(1):115-132

2 Eissenberg LG, Goldman WE, Schlesinger PH. Histoplasma capsulatum modulates the acidification of phagolysosomes. J Exp Med 1993;177(6):1605-1611

3 Sebghati TS, Engle JT, Goldman WE. Intracellular parasitism by Histoplasma capsulatum: fungal virulence and calcium dependence. Science 2000;290(5495):1368-1372

4 Trofa D, Nosanchuk JD. Histoplasmosis of the central nervous system. J Neuroparasitology 2012;3:1-7 
5 Veeravagu A, Ludwig C, Camara-Quintana JQ Jiang B, Lad N, Shuer L. Fungal infection of a ventriculoperitoneal shunt: histoplasmosis diagnosis and treatment. World Neurosurg 2013;80(1-2):222. e5-222.e13

6 Goldman M, Zackin R, Fichtenbaum CJ, et al; AIDS Clinical Trials Group A5038 Study Group. Safety of discontinuation of maintenance therapy for disseminated histoplasmosis after immunologic response to antiretroviral therapy. Clin Infect Dis 2004;38(10): 1485-1489

7 McKinsey DS, Spiegel RA, Hutwagner L, et al. Prospective study of histoplasmosis in patients infected with human immunodeficiency virus: incidence, risk factors, and pathophysiology. Clin Infect Dis 1997;24(6):1195-1203

8 Wheat LJ, Musial CE, Jenny-Avital E. Diagnosis and management of central nervous system histoplasmosis. Clin Infect Dis 2005;40(6): 844-852

9 Black KE, Baden LR. Fungal infections of the CNS: treatment strategies for the immunocompromised patient. CNS Drugs 2007;21(4):293-318

10 Saccente M. Central nervous system histoplasmosis. Curr Treat Options Neurol 2008;10(3):161-167

11 Carod-Artal F, Venturini M, Gomes E, de Mello M. Chronic central nervous system histoplasmosis in an immunocompetent patient [in Spanish]. Neurologia 2008;23(4):263-268

12 Hamada M, Tsuji S. Central nervous system histoplasmosis [in Japanese]. Brain Nerve 2009;61(2):129-134

13 Vullo V, Mastroianni CM, Ferone U, et al. Central nervous system involvement as a relapse of disseminated histoplasmosis in an Italian AIDS patient. J Infect 1997;35(1):83-84

14 Guarner J, Brandt ME. Histopathologic diagnosis of fungal infections in the 21st century. Clin Microbiol Rev 2011;24(2):247-280

15 Aravalli RN, Hu S, Woods JP, Lokensgard JR. Histoplasma capsulatum yeast phase-specific protein Yps3p induces Toll-like receptor 2 signaling. J Neuroinflammation 2008;5:30

16 Khasawneh FA, Ahmed S, Halloush RA. Progressive disseminated histoplasmosis presenting with cachexia and hypercalcemia. Int J Gen Med 2013;6:79-83

17 Nguyen FN, Kar JK, Zakaria A, Schiess MC. Isolated central nervous system histoplasmosis presenting with ischemic pontine stroke and meningitis in an immune-competent patient. JAMA Neurol 2013;70(5):638-641

18 Ramireddy S, Wanger A, Ostrosky L. An instructive case of CNS histoplasmosis in an immunocompetent host. Med Mycol Case Rep 2012;1(1):69-71

19 Cleary JD, Pearson M, Oliver J, Chapman SW. Association between Histoplasma exposure and stroke. J Stroke Cerebrovasc Dis 2008; 17(5):312-319

20 Paphitou NI, Barnett BJ. Solitary parietal lobe histoplasmoma mimicking a brain tumor. Scand J Infect Dis 2002;34(3):229-232
21 Loughan AR, Perna R, Hertza J. Cognitive impairment and memory loss associated with histoplasmosis: a case study. Clin Neuropsychol 2014;28(3):514-524

22 Shapiro JL, Lux JJ, Sprofkin BE. Histoplasmosis of the central nervous system. Am J Pathol 1955;31(2):319-335

23 Azizirad O, Clifford DB, Groger RK, Prelutsky D, Schmidt RE. Histoplasmoma: isolated central nervous system infection with Histoplasma capsulatum in a patient with AIDS. Case report and brief review of the literature. Clin Neurol Neurosurg 2007;109(2): 176-181

24 Hott JS, Horn E, Sonntag VKH, Coons SW, Shetter A. Intramedullary histoplasmosis spinal cord abscess in a nonendemic region: case report and review of the literature. J Spinal Disord Tech 2003; 16(2):212-215

25 John AM, Vasanthi N, Khurana A, et al. An unusual cause for rings in the brain. Hong Kong Med J 2012;18(4):346-347

26 Klein CJ, Dinapoli RP, Temesgen Z, Meyer FB. Central nervous system histoplasmosis mimicking a brain tumor: difficulties in diagnosis and treatment. Mayo Clin Proc 1999;74(8): 803-807

27 Murata M, Furusyo N, Otaguro S, Nabeshima S, Ariyama I, Hayashi J HIV infection with concomitant cerebral toxoplasmosis and disseminated histoplasmosis in a 45-year-old man. J Infect Chemother 2007;13(1):51-55

28 Parihar A, Tomar V, Ojha BK, Husain N, Gupta RK. Magnetic resonance imaging findings in a patient with isolated histoplasma brain abscess. Arch Neurol 2011;68(4):534-535

29 Saccente M, McDonnell RW, Baddour LM, Mathis MJ, Bradsher RW. Cerebral histoplasmosis in the azole era: report of four cases and review. South Med J 2003;96(4):410-416

30 Smith JS, Quiñones-Hinojosa A, Phillips JJ, Bollen AW, McDermott MW, Cha S. Limitations of diffusion-weighted imaging in distinguishing between a brain tumor and a central nervous system histoplasmoma. J Neurooncol 2006;79(2):217-218

31 Kastrup O, Wanke I, Maschke M. Neuroimaging of infections. NeuroRx 2005;2(2):324-332

32 Rangel-Castilla L, Hwang SW, White AC, Zhang YJ Neuroendoscopic diagnosis of central nervous system histoplasmosis with basilar arachnoiditis. World Neurosurg 2012; 77(2):E9-E13

33 Wheat LJ, Connolly P, Smedema M, et al. Activity of newer triazoles against Histoplasma capsulatum from patients with AIDS who failed fluconazole. J Antimicrob Chemother 2006;57(6): 1235-1239

34 Wheat LJ, Freifeld AG, Kleiman MB, et al; Infectious Diseases Society of America. Clinical practice guidelines for the management of patients with histoplasmosis: 2007 update by the Infectious Diseases Society of America. Clin Infect Dis 2007;45(7): 807-825 